\title{
(2) OPEN ACCESS \\ Are mesenchymal stem cells and derived extracellular vesicles valuable to halt the COVID-19 inflammatory cascade? Current evidence and future perspectives
}

\author{
Marta Monguió-Tortajada, ${ }^{1,2}$ Antoni Bayes-Genis, ${ }^{1,2,3,4}$ Antoni Rosell, ${ }^{3,5,6}$ \\ Santiago Roura ${ }^{1,2}$
}

${ }^{1}$ ICREC Research Program, Health Science Research Institute Germans Trias i Pujol (IGTP), Badalona, Catalunya, Spain

${ }^{2}$ CIBERCV, Instituto de Salud Carlos III, Madrid, Spain ${ }^{3}$ Department of Medicine, Autonomous University of Barcelona (UAB), Barcelona, Catalunya, Spain

${ }^{4}$ Cardiology Service, Germans Trias i Pujol University Hospital Badalona, Catalunya, Spain ${ }^{5}$ Servei de Pneumologia, Germans Trias i Pujol University Hospital, Badalona, Catalunya, Spain

${ }^{6}$ CIBERES, Instituto de Salud Carlos III, Madrid, Spain

\section{Correspondence to}

Dr Santiago Roura, ICREC, Fundació Institut d'Investigació en Ciències de la Salut Germans Trias i Pujol, Badalona 08916, Catalunya, Spain: sroura@gmail.com

Received 4 July 2020 Accepted 28 August 2020 Published Online First 15 December 2020

\section{Check for updates}

(c) Author(s) (or their employer(s)) 2021. Re-use permitted under CC BY-NC. No commercial re-use. See rights and permissions. Published by BMJ.

To cite: Monguió-

Tortajada M, Bayes-Genis A, Rosell A, et al. Thorax 2021:76:196-200.
Clinical management of patients suffering from COVID-19 includes infection prevention, control measures and supportive care, including supplemental oxygen and mechanical ventilatory support. ${ }^{12}$ If not expertly and individually managed with consideration for vasocentric features, ${ }^{3}$ 20\% of COVID-19 patients who exhibit acute respiratory distress syndrome (ARDS; $14.8 \%$ of hospitalised patients ${ }^{4}$ ) may eventually progress to multiorgan failure and death, even when not of advanced age or predisposed by pre-existing comorbidities or chronic diseases. ${ }^{5}$ In these patients, infection by SARS-CoV-2 activates both the innate and adaptive immune responses, provoking the production of large amounts of proinflammatory cytokines and chemokines, resulting in a clinical presentation (ie, unremitting high fever, lymphadenopathy, hepatosplenomegaly, cytopenia, hyperferritinaemia, central nervous system abnormalities, hypoalbuminaemia and capillary leak) similar to other systemic, uncontrolled cytokine release syndromes (CRS)(figure 1). ${ }^{67}$ Thus, at present, management of the potential inflammatory complications of COVID-19 by using appropriate immunosuppressive and immunomodulatory drugs is being explored. ${ }^{8}$

To date, a variety of novel and repurposed drugs with multiple pharmacological effects and therapeutic efficacies have been included in the potential armamentarium against COVID-19. ${ }^{9} 10$ For instance, among the antivirals preliminary tested, remdesivir has showing the best efficacy, with significant improvements in shortening the time to recovery and evidence of lower respiratory tract infection. ${ }^{11}$ It was issued an emergency use authorisation on 1 May 2020 for the treatment of COVID-19 patients hospitalised with severe disease by the Food and 
Drug Administration (FDA), and on 3 July 2020, it became the first treatment against COVID-19 with a conditional marketing authorisation for patients over 12 years old with pneumonia who require supplemental oxygen by the European Medicines Agency. Moreover, some researchers have promoted the antimalarial 4-aminoquinolines, chloroquine and hydroxychloroquine (HCQ), for the blocking of the progression to the dangerous ARDS phase of the disease due to their immunomodulatory effects. ${ }^{9}$ However, both drugs, even the less toxic HCQ, have been removed as therapeutic options for treating COVID-19 patients as they do not lead to clinical improvement. Indeed, the FDA recently revoked emergency use ruling for HCQ. Since large amounts of secreted interleukin 6 (IL-6) has been correlated to disease severity, administration of the humanised anti-IL- 6 receptor antibody inhibiting IL-6 tocilizumab is being tested in patients with severe SARS-CoV-2 infection complicated with CRS and ARDS. Published data from some of these clinical trials have revealed that response to tocilizumab is rapid, sustained and associated with significant clinical improvements, including reduction of lethality rate at 30 days. ${ }^{12-14}$ On the other hand, CRS also comprises massive IL-1 secretion and other cytokines. There are a number of ongoing studies using the recombinant human IL-1 receptor antagonist anakinra to clinically block the proinflammatory effects of IL- $1 \alpha / \beta$ against COVID-19. ${ }^{15}$ Preliminary findings by Huet et al and Cavalli et al are promising due treatment with anakinra reduces both the need for invasive mechanical ventilation and mortality in patients with severe forms of COVID-19, without serious life-threatening sideeffects. ${ }^{16}{ }^{17}$ Based on its ability to inhibit IL-1 production, colchicine is also an anti-inflammatory and immunomodulatory agent which is currently being tested in the COLCORONA (Colchicine Coronavirus SARS-CoV-2) trial (NCT04322682). Moreover, there is no sufficient evidence supporting tumour necrosis factor (TNF)- $\alpha$ or Janus kinase inhibition (etanercept and baricitinib, respectively, used for rheumatoid arthritis), or non-steroidal antiinflammatory agents, especially ibuprofen, as potential treatment options for COVID-19. ${ }^{18-21}$ Of note, the corticoid dexamethasone, which is used to treat common conditions such as arthritis, blood, hormone and immune system disorders, allergic reactions, certain skin and eye conditions, is the first anti-inflammatory drug to exert very significant clinical and mortality improvement in hospitalised patients with severe respiratory complications of COVID-19, as reported by the RECOVERY (Randomised Evaluation of COVID-19 thERapY) investigators. ${ }^{22}$ The study concluded that treatment with dexamethasone at a dose of $6 \mathrm{mg}$ once daily for up to 10 days reduced 28-day mortality in patients who were receiving respiratory support, but induced no benefit or even possibility of harm among those who did not require oxygen.

Alternatively, treatment with mesenchymal stromal or stem cells (MSCs) has been proposed as a promising therapeutic strategy to halt the uncontrolled inflammatory cascade and, at the same time, reduce lung fibrosis and abnormal pulmonary function occurring post-COVID-19 $9^{23} 24$ (figure 1). In particular, an incidence of lung fibrosis in approximately $7 \%$ to $10 \%$ of survivors, similar to other ARDS conditions and to the pathogenesis found in MERS and SARS, could be estimated from compelling data. ${ }^{42}$ MSCs represent a heterogeneous population of non-haematopoietic multipotent stromal cells with a well-established, specific cell surface expression pattern, low alloreactivity (ie, low expression of major histocompatibility complex (MHC)-I and lack of MHC-II and co-stimulatory molecules) and the ability to differentiate tissues of mesodermal lineages. ${ }^{25}$ Despite the remarkable heterogeneity of MSC sources and the lack of specific biomarkers predicting the success of prospective MSC-based therapeutic products, MSCs show powerful immunomodulatory, anti-oxidant and angiogenic capabilities once implanted in vivo. ${ }^{26}$ For example, MSCs induce a marked shift in the pro-inflammatory pulmonary microenvironment induced by paraquat poisoning, ventilator-induced injury or infectious models of lung injury. ${ }^{27} 28$ Particularly, the beneficial effects of MSCs are shown to be associated with the reduction in TNF- $\alpha$, IL- 1 and IL- 6 through the release of hepatocyte growth factor (HGF), prostaglandin-E2 ( $\left.\mathrm{PGE}_{2}\right)$, lipoxin A4 (LXA4) and TNF-stimulated gene 6 protein (TSG-6), suppression of inflammatory $\mathrm{T}$-cell proliferation by indoleamine 2,3-dioxygenase expression, switch from Th1 and Th17 responses to Th2, and inhibition of monocytes and myeloid dendritic cells maturation (figure 2). MSCs induce M2 polarisation by contact-dependent and paracrine factors such as HGF, PGE 2 and TSG-6, resulting in a monocyteaided increase in anti-inflammatory IL-10, which synergistically induces regulatory $\mathrm{T}$ cells and switches on tissue repair mechanisms. Furthermore, MSCs interfere in different pathways that promote lung fibrosis resulting in protective effects, as demonstrated in several lung injury models. ${ }^{27}$ Indeed, MSC delivery also reduces collagen-rich scarring and pulmonary fibrosis Ashcroft score by inhibiting the expression of TGF- $\beta$, IFN- $\gamma$ and TNF- $\alpha$, balancing extracellular matrix remodelling enzymes (matrix metalloproteinase (MMPs) and tissue inhibitor of MMPs), collagen deposition and impairing myofibroblasts differentiation through HGF and TSG-6 in rodent models of bleomycin-induced acute lung injury. ${ }^{29-31}$ Moreover, MSCs have the potential to release antimicrobial peptides such as LL37, also stimulating the phagocytic capacity of macrophages together with the anti-apoptotic HGF secretion. ${ }^{32}$ MSCs can protect against infection-associated alveolar damage such as influenza A (H5N1) virus infection, and prevent or reduce acute lung injury in vivo. ${ }^{33}$ Specifically, MSCs reduce the injury-related alveolar oedema and endothelial permeability by the release of keratinocyte growth factor (KGF) to enhance the sodium-dependent alveolar fluid clearance through epithelial sodium channel $(\mathrm{ENaC})$, and have anti-apoptotic and anti-oxidative mechanisms to restore cytokine-damaged alveolar type II cells and epithelial and endothelial injury through the release of angiopoietin-1, lipoxin A4 (LXA4) and TSG-6. In fact, the successful use of allogeneic MSC therapy in influenza (H7N9)-induced ARDS has been recently documented with an open-label controlled trial showing significantly lower mortality in the treatment group $(17.6 \%)$ to the control group $(54.5 \%),{ }^{34}$ and proving safe in a phase IIa clinical trial for ARDS. ${ }^{35}$ In light of these findings, although there is no clinical evidence published on ARDS-associated lung fibrosis reduction after MSC therapy per se, MSCs modulate apoptosis and oxidative injury, avoid inflammation, have antifibrotic properties, reduce fibroblasts activation and, thus, enhance lung repair. This could have a positive impact in COVID-19 patients if MSCs are used to treat ARDS in the early stages of SARS-CoV-2 infection.

For that, numerous clinical studies have begun to probe whether treatment with human MSCs from distinct origins improves the course and prognosis of COVID-19 patients with no adverse effects. $^{3637}$ In this context, Zhang and colleagues have reported data from the intravenous infusion of MSCs from human umbilical cord Wharton's jelly (WJ-MSCs) in a single-patient study. ${ }^{38}$ Celltreated patient, who already received antiviral, anti-inflammatory and antipyretic therapy and displayed a $\mathrm{C}$ reactive protein of $<602$ weeks before cell infusion, experienced disappearance of both fever and shortness of breath on the second day. Chest CT imaging also showed that the ground-glass opacity and pneumonia infiltration had extensively diminished on the sixth day after transplantation of WJ-MSCs. Additional analyses revealed that COVID-19 lymphopenia improved, with increased counts of $\mathrm{T}$ lymphocytes (both $\mathrm{CD}^{+}$and $\mathrm{CD}^{+}$), and the level of pro-inflammatory IL-6 


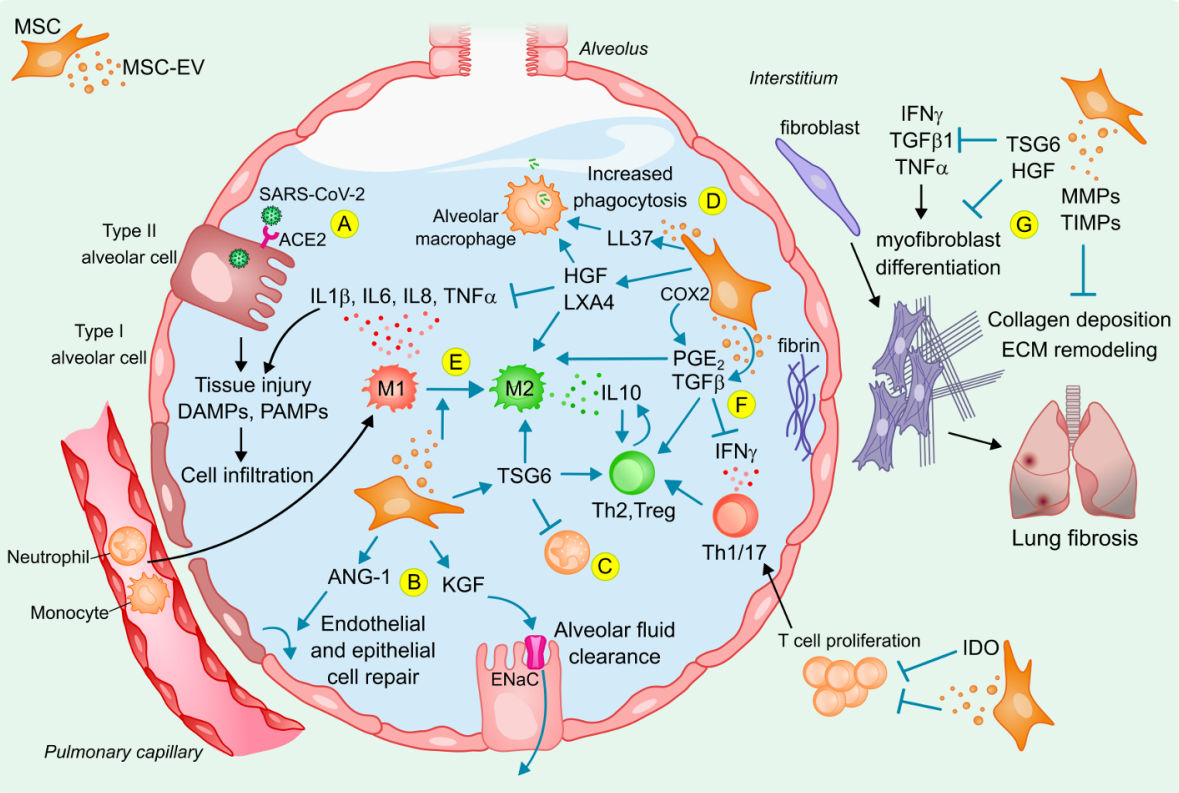

Figure 2 Potential mechanisms of action of MSCs and derived EVs to reduce lung injury in acute respiratory distress syndrome following SARSCoV-2 infection. (A) SARS-CoV-2 infection causes cell and tissue injury, release of DAMPs, PAMPs and inflammatory mediators that are sensed by neighbouring cells and alveolar macrophages that amplify an inflammatory response in the alveolus, promoting immune cell infiltration and accumulation of protein-rich alveolar oedema fluid. Infused MSCs and derived EVs can reduce inflammation and trigger tissue repair by (B) increasing alveolar fluid clearance and have anti-apoptotic and anti-oxidative effects to restore injured alveolar, epithelial and endothelial cells; (C) reducing neutrophil activation, which also decreases inflammatory cell infiltration; (D) enhancing phagocytosis and viability of alveolar macrophages; (E) reducing inflammation and inducing M2 polarisation of monocytes that results in IL-10 production to induce (F) regulatory T cells and Th2 switch from Th1 and Th17 responses, together with a modulation of T-cell proliferation; (G) protecting from lung fibrosis. ANG-1, angiopoietin-1; COX2, cyclooxygenase-2; DAMPs, danger-associated molecular patterns; ECM, extracellular matrix; ENaC, epithelial sodium channel; $\mathrm{HGF}$, hepatocyte growth factor; IDO, indoleamine 2,3-dioxygenase; IFN $\gamma$, interferon gamma; IL, interleukin; KGF, keratinocyte growth factor; LL37, antimicrobial peptide $\beta$-cathelicidin; LXA4, lipoxin A4; MMP, matrix metalloproteinase; MSC, mesenchymal stem cell; MSC-EV, MSC-derived extracellular vesicles; PAMPs, pathogen-associated molecular patterns; PGE2, prostaglandin E2; TGF $\beta$, transforming growth factor beta; TIMP, tissue inhibitor of MMP; TNF- $\alpha$, tumour necrosis factor alpha; Treg, regulatory T-cell; TSG-6, TNF-stimulated gene 6 protein.

and TNF- $\alpha$ cytokines, and $\mathrm{C}$ reactive protein was significantly decreased after cell treatment. Leng et al reported a clinical trial pilot study treating seven COVID-19 patients (two common, four severe and one critically severe case) with MSCs. Post-treatment, pulmonary function improved within 2 days, the levels of TNF- $\alpha$, $\mathrm{C}$ reactive protein and overactivated lymphocytes decreased significantly, while levels of IL-10, regulatory dentritic cell populations and peripheral lymphocytes increased. They concluded that the intravenous infusion of MSCs improved the functional outcome of patients with COVID-19 pneumonia, especially patients whose condition was critically severe, without any adverse effects. ${ }^{39}$ More recently, 13 COVID-19 adult patients under invasive mechanical ventilation who had received previous antiviral and/or anti-inflammatory treatments were infused with allogeneic adipose tissue-derived MSCs. ${ }^{40}$ MSC treatment was followed by a reduction in $\mathrm{C}$ reactive protein, IL-6, ferritin, $\mathrm{LDH}$ and $\mathrm{D}$-dimer), especially in those patients with clinical improvement (70\%). Also, no adverse events related to cell therapy were observed. Remarkably, ongoing industry trials such as those conducted by Mesoblast and Athersys are greatly expected to confirm initial encouraging results from the evaluation of novel efficient MSC-based products for ARDS treatment. ${ }^{41}{ }^{42}$ Collectively, the preliminary published reports support that administration of MSCs is safe, alleviates the aggressive inflammatory status and is followed by clinical and biological improvement in most COVID-19 patients.

In addition, a number of researchers devoted to the development of MSC-based therapies for years have turned their attention to derivatives prepared from MSC-conditioned media, referred to as extracellular vesicles (EVs). This foundation arises from growing research showing that MSC-EVs are harmless and trigger, at least, similar effects as their parent cells in acute and chronic lung injury, sepsis and ARDS models. ${ }^{43} 44$ Thus, MSC-EVs could be more valuable than the MSC themselves to halt COVID-19 inflammatory cascade. Specifically, a better and safer refining of the paracrine function of therapeutic MSCs could enhance therapy outcomes for COVID-19 and, in turn, avoid some potential side-effects from MSC administration. But what are the scientific fundamentals underlying this alternative therapy? First, the fact that a minimum amount of retained cells exert beneficial effects points out that the contribution of MSCs is mainly driven by paracrine signalling, rather than a direct cell-to-cell effect in vivo. In line, increasing data suggests that paracrine activity of MSCs is accompanied by their well-determined production and secretion of $\mathrm{EVs}$, which promote similar functional properties as the parental cells. ${ }^{45} \mathrm{EVs}$, which constitute stable, lipid bilayer-encapsulated nanostructures and specifically transfer intercellular proteins, lipids and non-coding nucleic acids through the circulation to tissues and/or cells at distal sites, act as great versatile immune regulators. In terms of advantages, non-replicative EVs are theoretically unaltered by microenvironmental factors (eg, the inflammatory milieu) since their double-leaflet lipid membranes protect the inner molecular cargo from degradation and guarantee entry into surrounding or distant targeted cells nor teratogenic, with low alloreactivity, besides easy handling and storage. Second, EVs may arrive easily at sites of the lungs getting damaged and scarred by pulmonary fibrosis for a targeted modulation of inflammation due to their characteristic 
nanosize which, in turn, omits the possibility of pulmonary thrombosis after intravascular MSC delivery as the majority of administered cells are initially trapped in the lungs. ${ }^{46}$ Several studies comprehensively described the thromboembolism effect of MSC infusion and potential thrombolytic regimens for safer applications and to maximise clinical benefit to the patients. ${ }^{478}$ In this regard, SARS-CoV-2 infection has been reported to cause damage to other parts of the body beyond the respiratory tract, such as the cardiovascular system, causing severe forms of myocarditis and endotheliitis in addition to the respiratory dysfunction. ${ }^{49}$ The blood vessels present high expression of ACE2 receptors and, thus, excessive blood clotting has been observed in COVID-19 patients as a direct consequence of the viral infection on the circulatory system. ${ }^{50}$ Particularly, a large amount of D-dimer, which is a product that indicates the presence of blood clots, is detected and consequently the vast majority of COVID-19 patients require further anticoagulant treatments. Furthermore, the infection by SARS-CoV-2 can cause a marked constriction of blood vessels that causes reduction in blood circulation and damage to peripheral tissues such as subcutaneous inflammatory affections, mainly in the trunk and extremities. ${ }^{51}$ Indeed, these circulatory alterations could be reinforced by MSC-based therapies due to some relevant issues that have been raised regarding the safety of MSC infusions and the thrombogenic risk they could provoke. The size of MSCs could be a significant cause of blood vessel obstructions favouring lethal pulmonary thromboembolism, as well as MSCs have been shown to exhibit a marked procoagulant activity linked to the expression of tissue factor that, in turn, can potentially initiate unwanted coagulation processes when in contact with blood. ${ }^{52}$ Some authors have also described a dual activation of both the coagulation and the complement pathway referred to as instant blood-mediated inflammatory reaction. ${ }^{53}$ Consequently, approaches to detect and prevent these unreported yet harmful MSCs' effects are under investigation, which could in turn give an explanation to the limited benefits and cell engraftment described in many clinical trials. On the contrary, among their multifaceted role in the modulation of biological responses, the involvement of MSC-EVs in angiogenesis-related diseases is gaining interest. ${ }^{54}$ Other important concerns should be taken into account in order to keep treating COVID-19 patients with MSCs or to inspire alternative clinical practice such as those based in EVs. As mentioned above, they include the pulmonary retention of intravenously administered MSCs, ${ }^{55} 56$ from where they secrete anti-inflammatory factors or agents with systemic effects (including EVs), and cell viability, which is crucial for the success of cell therapies, ${ }^{35}$ a drawback that EVs could greatly circumvent.

Of concern, together with the ongoing clinical trials using MSCs in COVID-19 that need to counteract limitations related to patient coagulopathy and thromboprophylaxis while probing cell product quality and delivery route to guarantee treatment safety and efficacy, the latest research news highlight a number of registered clinical trials evaluating the potential benefit of MSC-EVs. ${ }^{57}$ However, leading voices for advancing clinical translation of cell and gene therapies worldwide such as International Society for Cell and Gene Therapy and the International Society for Extracellular Vesicles have rapidly launched their recommendations on providing 'appropriate manufacturing and quality control provisions, pre-clinical safety and efficacy data, rationale clinical trial design, and proper regulatory oversight' of EV-based therapeutics for COVID-19 before administration to patients. ${ }^{58}$

In conclusion, since the first cases reported in Wuhan, China, COVID-19 accounts for more than 68000000 infections and over 1500000 deaths worldwide. This has led to declare the COVID-19 pandemic as a real global health emergency for the WHO. Although COVID-19 infection may be asymptomatic or cause only mild symptoms in the majority of the cases, it provokes interstitial pneumonia, CRS and ARDS in nearly $15 \%$ of the cases, especially in people who are old and have co-morbidities. In general, treatments with the focus on modulating this exacerbated inflammation may thus improve clinical outcomes, and ultimately improve disease death rate. For that, a battery of anti-inflammatory drugs has been tested to limit exacerbated immune response in severe patients with yet modest results. Alternatively, more compatible, biological therapies, such as those based on MSCs, are gaining interest to mitigate ARDS. In this context, meanwhile, the numerous ongoing clinical trials of MSCs for COVID-19 will determine whether adoptive MSC transfer therapy is an ideal choice for treatment; their secreted EVs are also envisioned as promising in terms of lack of risk of thrombosis, inalterability over time, low alloreactivity and better usage and biodistribution. Indeed, EV administration should be appropriately manufactured, and pre-clinically and clinically tested following rational clinical trial design for rigorous and meaningful safety and efficacy data.

Contributors MM-T, AB-G, AR and SR approved the final manuscript as submitted and are accountable for all aspects of the work, including conception and design, and writing of the manuscript. In specific, MM-T also conceived and created schematic images.

Funding This work was supported in part by the PERIS (SLT002/16/00234) and SGR programmes (2017-SGR-483) from the Generalitat de Catalunya, 'La Caixa' Banking Foundation, grants from the Spanish Ministry of Economy and CompetitivenessMINECO (SAF2017-84324-C2-1-R and PID-2019-11137RB-100), Instituto de Salud Carlos III (PIC18/00014) funds, Red de Terapia Celular-TerCel (RD16/00111/0006), CIBER Cardiovascular (CB16/11/00403) CIBER Enfermedades Respiratorias (CB06/06/1089) projects, as part of the Plan Nacional de I+D+l, and co-funded by ISCIII-Subdirección General de Evaluación y el Fondo Europeo de Desarrollo Regional (FEDER), and by the Call for Expression of Interest (Eol) for Collaborative Projects on Regenerative Medicine 2019 of Centre for Regenerative Medicine of Barcelona (CMR(B)).

Competing interests None declared.

\section{Patient consent for publication Not required.}

Provenance and peer review Not commissioned; externally peer-reviewed.

Open access This is an open access article distributed in accordance with the Creative Commons Attribution Non Commercial (CC BY-NC 4.0) license, which permits others to distribute, remix, adapt, build upon this work non-commercially, and license their derivative works on different terms, provided the original work is properly cited, appropriate credit is given, any changes made indicated, and the use is non-commercial. See: http://creativecommons.org/licenses/by-nc/4.0/.

\section{REFERENCES}

1 Grasselli G, Zangrillo A, Zanella A, et al. Baseline characteristics and outcomes of 1591 patients infected with SARS-CoV-2 admitted to ICUs of the Lombardy region, Italy. JAMA 2020;323:1574-81.

2 Marini JJ, Gattinoni L. Management of COVID-19 respiratory distress. JAMA 2020:323:2329.

3 Ackermann M, Verleden SE, Kuehnel M, et al. Pulmonary vascular Endothelialitis, thrombosis, and angiogenesis in Covid-19. N Engl J Med Overseas Ed 2020;383:120-8

4 Sun P, Qie S, Liu Z, et al. Clinical characteristics of hospitalized patients with SARSCoV-2 infection: a single arm meta-analysis. J Med Virol 2020;92:612-7.

5 Moore JB, June $\mathrm{CH}$. Cytokine release syndrome in severe COVID-19. Science 2020:368:473-4.

6 Gao Y-M, Xu G, Wang B, et al. Cytokine storm syndrome in coronavirus disease 2019: a narrative review. J Intern Med 2020. doi:10.1111/joim.13144. [Epub ahead of print: 22 Jul 2020]

7 England JT, Abdulla A, Biggs CM, et al. Weathering the COVID-19 storm: lessons from hematologic cytokine syndromes. Blood Rev 2020;100707:100707.

8 Stebbing J, Phelan A, Griffin I, et al. COVID-19: combining antiviral and antiinflammatory treatments. Lancet Infect Dis 2020;20:400-2. 
9 Soy M, Keser G, Atagündüz P, et al. Cytokine storm in COVID-19: pathogenesis and overview of anti-inflammatory agents used in treatment. Clin Rheumatol 2020;39:2085-94

10 Choudhary S, Malik YS, Tomar S. Identification of SARS-CoV-2 Cell Entry Inhibitors by Drug Repurposing Using in silico Structure-Based Virtual Screening Approach. Front Immunol 2020;11:1664.

11 Beigel JH, Tomashek KM, Dodd LE, et al. Remdesivir for the treatment of Covid-19 final report. N Engl J Med Overseas Ed 2020.

12 Xu X, Han M, Li T, et al. Effective treatment of severe COVID-19 patients with tocilizumab. Proc Natl Acad Sci U S A 2020;117:10970-5.

13 Toniati P, Piva S, Cattalini M, et al. Tocilizumab for the treatment of severe COVID-19 pneumonia with hyperinflammatory syndrome and acute respiratory failure: a single center study of 100 patients in Brescia, Italy. Autoimmun Rev 2020;19:102568.

14 Perrone F, Piccirillo MC, Ascierto PA, et al. Tocilizumab for patients with COVID-19 pneumonia. The TOCIVID-19 prospective phase 2 trial. MedRxiv 2020.

15 U.S. National library of medicine shares information about clinical trials using Anakinra against COVID-19 either in recruitment or pre-recruitment stage. Available: https://clinicaltrials.gov/ct2/results? cond $=$ Covid19\&term =anakinra\&cntry $=\&$ state $=\&$ city $=\&$ dist $=[$ Accessed 19 Aug 2020].

16 Huet T, Beaussier H, Voisin O, et al. Anakinra for severe forms of COVID-19: a cohort study. Lancet Rheumatol 2020;2:e393-400.

17 Cavalli G, De Luca G, Campochiaro C, et al. Interleukin-1 blockade with highdose anakinra in patients with COVID-19, acute respiratory distress syndrome, and hyperinflammation: a retrospective cohort study. Lancet Rheumatol 2020;2:e325-31.

18 Mehta P, McAuley DF, Brown M, et al. COVID-19: consider cytokine storm syndromes and immunosuppression. Lancet 2020;395:1033-4.

19 Stebbing J, Phelan A, Griffin I, et al. COVID-19: combining antiviral and antiinflammatory treatments. Lancet Infect Dis 2020;20:400-2.

20 Wang $M$, Zhou Y, Zong Z, et al. A precision medicine approach to managing 2019 novel coronavirus pneumonia. Precis Clin Med 2020;3:14-21.

21 Day M. Covid-19: ibuprofen should not be used for managing symptoms, say doctors and scientists. BMJ 2020;368:m1086.

22 RECOVERY Collaborative Group, Horby P, Lim WS, et al. Dexamethasone in Hospitalized Patients with Covid-19 - Preliminary Report. N Engl J Med 2020. doi:10.1056/NEJMoa2021436. [Epub ahead of print: 17 Jul 2020].

23 Mo X, Jian W, Su Z, et al. Abnormal pulmonary function in COVID-19 patients at time of hospital discharge. Eur Respir J 2020;55:2001217.

24 Thille AW, Esteban A, Fernández-Segoviano P, et al. Chronology of histological lesions in acute respiratory distress syndrome with diffuse alveolar damage: a prospective cohort study of clinical autopsies. Lancet Respir Med 2013;1:395-401.

25 Dominici M, Le Blanc K, Mueller I, et al. Minimal criteria for defining multipotent mesenchymal stromal cells. The International Society for cellular therapy position statement. Cytotherapy 2006;8:315-7.

26 Carreras-Planella L, Monguió-Tortajada M, Palma E. Stem cells: Immunotherapy in solid organ transplantation. Encyclopedia of Tissue Engineering and Regenerative Medicine, Elsevier, 2019: 81-90.

27 Mclntyre LA, Moher D, Fergusson DA, et al. Efficacy of mesenchymal stromal cell therapy for acute lung injury in preclinical animal models: a systematic review. PLOS One 2016:11:e0147170.

28 Laffey JG, Matthay MA. Fifty years of research in ARDS. cell-based therapy for acute respiratory distress syndrome. biology and potential therapeutic value. Am J Respir Crit Care Med 2017;196:266-73.

29 Moodley Y, Atienza D, Manuelpillai U, et al. Human umbilical cord mesenchymal stem cells reduce fibrosis of bleomycin-induced lung injury. Am J Pathol 2009;175:303-13.

30 Ortiz LA, Dutreil M, Fattman C, et al. Interleukin 1 receptor antagonist mediates the antiinflammatory and antifibrotic effect of mesenchymal stem cells during lung injury. Proc Natl Acad Sci U S A 2007;104:11002-7.

31 Srour N, Thébaud B. Mesenchymal stromal cells in animal bleomycin pulmonary fibrosis models: a systematic review. Stem Cells Trans/ Med 2015;4:1500-10.

32 Gupta N, Krasnodembskaya A, Kapetanaki M, et al. Mesenchymal stem cells enhance survival and bacterial clearance in murine Escherichia coli pneumonia. Thorax 2012:67:533-9.

33 Chan MCW, Kuok DIT, Leung $\mathrm{CYH}$, et al. Human mesenchymal stromal cells reduce influenza A H5N1-associated acute lung injury in vitro and in vivo. Proc Natl Acad Sci USA 2016:113:3621-6.

34 Chen J, Hu C, Chen L, et al. Clinical study of mesenchymal stem cell treating acute respiratory distress syndrome induced by epidemic influenza $A$ (H7N9) infection, a hint for COVID-19 treatment. Engineering 2020. doi:10.1016/j.eng.2020.02.006. [Epub ahead of print: 28 Feb 2020].
35 Matthay MA, Calfee CS, Zhuo H, et al. Treatment with allogeneic mesenchymal stromal cells for moderate to severe acute respiratory distress syndrome (start study): a randomised phase 2A safety trial. Lancet Respir Med 2019;7:154-62.

36 Golchin A, Seyedjafari E, Ardeshirylajimi A. Mesenchymal stem cell therapy for COVID-19: present or future. Stem Cell Rev Rep 2020:1-7

37 Khoury M, Cuenca J, Cruz FF, et al. Current status of cell-based therapies for respiratory virus infections: applicability to COVID-19. Eur Respir J 2020;55:2000858.

38 Zhang Y, Ding J, Ren S, et al. Intravenous infusion of human umbilical cord Wharton's jelly-derived mesenchymal stem cells as a potential treatment for patients with COVID-19 pneumonia. Stem Cell Res Ther 2020;11:207.

39 Leng Z, Zhu R, Hou W, et al. Transplantation of ACE2 Mesenchymal Stem Cells Improves the Outcome of Patients with COVID-19 Pneumonia. Aging Dis 2020;11:216-28.

40 Sánchez-Guijo F, García-Arranz M, López-Parra M, et al. Adipose-Derived mesenchymal stromal cells for the treatment of patients with severe SARS CoV-2 pneumonia requiring mechanical ventilation. A proof of concept study. EClinicalMedicine 2020;25:100454

41 Mesoblast shares information about its clinical trials, including that devoted to mesenchymal stem cells for the treatment of moderate to severe COVID-19 acute respiratory distress syndrome (NCT04371393). Available: https://www.mesoblast com/product-candidates/clinical-trials" https://www.mesoblast.com/productcandidates/clinical-trials [Accessed 18 Aug 2020].

42 Athersys Inc. Shares information about the MACOVIA study (MultiStem administration for COVID-19 induced ARDS). Available: https://www.athersys.com/clinical-trials/ ards/" https://www.athersys.com/clinical-trials/ards/ [Accessed 18 Aug 2020].

43 Worthington EN, Hagood JS. Therapeutic use of extracellular vesicles for acute and chronic lung disease. Int J Mol Sci 2020;21:2318

44 Mahida RY, Matsumoto S, Matthay MA. Extracellular vesicles: a new frontier for research in acute respiratory distress syndrome. Am J Respir Cell Mol Biol 2020;63:15-24

45 Monguió-Tortajada M, Roura S, Gálvez-Montón C, et al. Nanosized UCMSC-derived extracellular vesicles but not conditioned medium exclusively inhibit the inflammatory response of stimulated T cells: implications for nanomedicine. Theranostics 2017;7:270-84.

46 Coppin L, Sokal E, Stéphenne X. Thrombogenic risk induced by intravascular mesenchymal stem cell therapy: current status and future perspectives. Cells 2019:8:1160.

47 Tatsumi K, Ohashi K, Matsubara Y, et al. Tissue factor triggers procoagulation in transplanted mesenchymal stem cells leading to thromboembolism. Biochem Biophys Res Commun 2013;431:203-9.

48 Wu Z, Zhang S, Zhou L, et al. Thromboembolism induced by umbilical cord mesenchymal stem cell infusion: a report of two cases and literature review. Transplant Proc 2017;49:1656-8.

49 Guzik TJ, Mohiddin SA, Dimarco A, et al. COVID-19 and the cardiovascular system: implications for risk assessment, diagnosis, and treatment options. Cardiovasc Res 2020;116:1666-87.

50 Varga Z, Flammer AJ, Steiger P, et al. Endothelial cell infection and endotheliitis in COVID-19. Lancet 2020:395:1417-8.

51 Klok FA, Kruip MJHA, van der Meer NJM, et al. Incidence of thrombotic complications in critically ill ICU patients with COVID-19. Thromb Res 2020;191:145-7.

52 Oeller M, Laner-Plamberger S, Hochmann S, et al. Selection of tissue factor-deficient cell transplants as a novel strategy for improving Hemocompatibility of human bone marrow stromal cells. Theranostics 2018:8:1421-34.

53 Hoogduijn MJ, Roemeling-van Rhijn M, Engela AU, et al. Mesenchymal stem cells induce an inflammatory response after intravenous infusion. Stem Cells Dev 2013;22:2825-35.

54 Todorova D, Simoncini S, Lacroix R, et al. Extracellular vesicles in angiogenesis. Circ Res 2017;120:1658-73.

55 Fischer UM, Harting MT, Jimenez F, et al. Pulmonary passage is a major obstacle for intravenous stem cell delivery: the pulmonary first-pass effect. Stem Cells Dev 2009;18:683-92.

56 Eggenhofer E, Benseler V, Kroemer A, et al. Mesenchymal stem cells are short-lived and do not migrate beyond the lungs after intravenous infusion. Front Immunol 2012:3:297.

57 Khoury M, Rocco PRM, Phinney DG, et al. Cell-Based therapies for coronavirus disease 2019: proper clinical investigations are essential. Cytotherapy 2020. doi:10.1016/j.jcyt.2020.04.089. [Epub ahead of print: 17 Apr 2020]

58 Börger V, Weiss DJ, Anderson JD, et al. ISEV and ISCT statement on EVs from MscS and other cells: considerations for potential therapeutic agents to suppress COVID-19. Cytotherapy 2020:22. 